\title{
EFFECTS OF CORE-HOLE SCREENING ON SPIN-POLARISED AUGER SPECTRA FROM FERROMAGNETIC Ni
}

\author{
T. Wegner, M. Potthoff and W. Nolting \\ Lehrstuhl "Festkörpertheorie", Institut für Physik, Humboldt-Universität zu Berlin \\ Invalidenstraße 110, 10115 Berlin, Germany
}

\begin{abstract}
We calculate the spin- and temperature-dependent local density of states for ferromagnetic $\mathrm{Ni}$ in the presence of a core hole at a distinguished site in the lattice. Correlations among the valence electrons and between valence and core electrons are described within a multi-band Hubbard model which is treated by means of second-order perturbation theory around the Hartree-Fock solution. The core-hole potential causes strong screening effects in the $\mathrm{Ni}$ valence band. The local magnetic moment is found to be decreased by a factor of 5-6. The consequences for the spin polarisation of CVV Auger electrons are discussed.
\end{abstract}

PACS numbers: 79.20.Fv, 71.20.Be, 75.60.Ej

It was pointed out by Allenspach et al. [1] that the experimentally observed spin polarisation of the CVV Auger spectrum of $\mathrm{Ni}$ is substantially smaller than the band spin-polarisation. Contrarily, in the case of Fe the band and Auger spin-polarisations are comparable. The authors of Ref. [1] argue that the ground-state configuration of $\mathrm{Ni}$ in a solid is $3 d^{9}$ which as a consequence of the core-hole screening becomes a $3 d^{10}$ configuration with a vanishing magnetic moment. The observed finite Auger polarisation is then attributed to a core-hole polarisation caused by resonant excitation of a core electron into the valence band. Furthermore, in the case of $\mathrm{Ni}$ only the minority-spin $(3 d)$ states are unoccupied (strong ferromagnet). While the situation is different in the case of $\mathrm{Fe}$, since there are unoccupied minority, as well as, majority spin states (weak ferromagnet).

In the present paper we investigate the effects of core-hole screening in the initial state of the Auger process quantitatively, i.e. in a model of correlated itinerant electrons. Besides the local magnetic moment we are interested in the local density of states, which is relevant for the Auger line shape as is already known from the simple self-convolution model of Lander [2].

We consider a multi-band Hubbard-type model for the $3 d, 4 s$, and $4 p$ electrons. The atomic basis orbitals are assumed to have a well-defined angular-momentum character $L=\{l, m\}$. The hopping and overlap integrals for the one-particle 
part of the Hamiltonian corresponding to the non-orthogonal atomic basis are taken from (paramagnetic) tight-binding band-structure calculations [3]. The relatively broad $4 s$ and $4 p$ bands are assumed to be sufficiently well described by band theory. The Coulomb interaction among the $3 d$ electrons is assumed to be strongly screened. Consequently, the interaction part of the Hamiltonian consists of on-site $3 d$ interactions only. Exploiting atomic symmetries, the complete Coulomb matrix $U_{L_{1} L_{2} L_{4} L_{3}}$ can be expressed via $3 j$-symbols [4] in terms of three effective Slater parameters $\left(F^{0}, F^{2}, F^{4}\right)$. Equivalently, the Coulomb matrix can be parametrised by the averaged direct and exchange correlation parameters $U$ and $J$ (we take the atomic ratio for the effective Slater integrals $F^{2} / F^{4}=0.625$ which is a reasonable assumption for $3 d$-transition metals [5]).

The one-particle excitation spectrum is calculated by second-order perturbation theory around the Hartree-Fock solution (SOPT-HF) [6]. We furthermore employ the local approximation since it is known [7] that the effects due to the (weak) $k$-dependence of the self-energy are fairly small within SOPT-HF applied to the multi-band Hubbard model. The interaction parameters are chosen as $U=2.47 \mathrm{eV}$ and $J=0.5 \mathrm{eV}$ which reproduce the measured value $m=0.56 \mu_{\mathrm{B}}$ for the $T=0$ magnetic moment [8]. The ratio $J / U \approx 0.2$ is a typical value for the late $3 d$-transition metals.

The resulting density of states for $\mathrm{Ni}(\mathrm{fcc})$ is shown on the l.h.s. of Fig. 1 for three different temperatures $\left(T=0, T=0.9 T_{\mathrm{C}}\right.$ and $\left.T=T_{\mathrm{C}}\right)$. Due to the imaginary part of the SOPT-HF self-energy the spectra are strongly damped compared to the results of band-structure calculations. In the energy region $\pm 2 \mathrm{eV}$ around the chemical potential $\mu$ there are distinguishable structures. As a consequence of the non-zero slope of the real part of the self-energy at $E=\mu$, a considerable band-narrowing is observed. The temperature-dependent difference between $\uparrow$ and $\downarrow$ spectra is more or less given by a rigid shift which disappears for $T \rightarrow T_{\mathrm{C}}$. The corresponding magnetisation curve (circles in the inset of Fig. 1) has a Brillouin-function-like form. The Curie temperature turns out to be $T_{\mathrm{C}}=1655 \mathrm{~K}$ and is thereby about a factor of 2.6 larger than the measured value of $624 \mathrm{~K}$ [9]. This overestimation of $T_{\mathrm{C}}$ is probably due to the mean-field character of the SOPT-HF. It should be noted that a simple LDA $+U$ (Hartree-Fock) calculation yields a much higher value $\left(T_{\mathrm{C}} \approx 2500 \mathrm{~K}\right)$.

Conceptually, the Auger process can be divided into two subprocesses. The first one is the creation of a core hole at a particular lattice site $\boldsymbol{R}_{\mathrm{c}}$, e.g. by absorbing an X-ray quantum. The second one is the radiationless decay of the core hole by ejecting an Auger electron. Provided that the lifetime of the core hole is large compared to typical relaxation times of the valence electrons, the two subprocesses become independent [10]. Since the Auger process takes place locally, the Auger spectrum is influenced by the additional core-hole potential in the initial state.

To describe the core-hole effects we have to extend the Hamiltonian. In the one-particle part we additionally consider a non-degenerate (s-like)and dispersionless core level with a one-particle energy well below the valence band. In the interaction part we add a density-density interaction between core and valence electrons. This interaction is responsible for the screening of the core-hole 


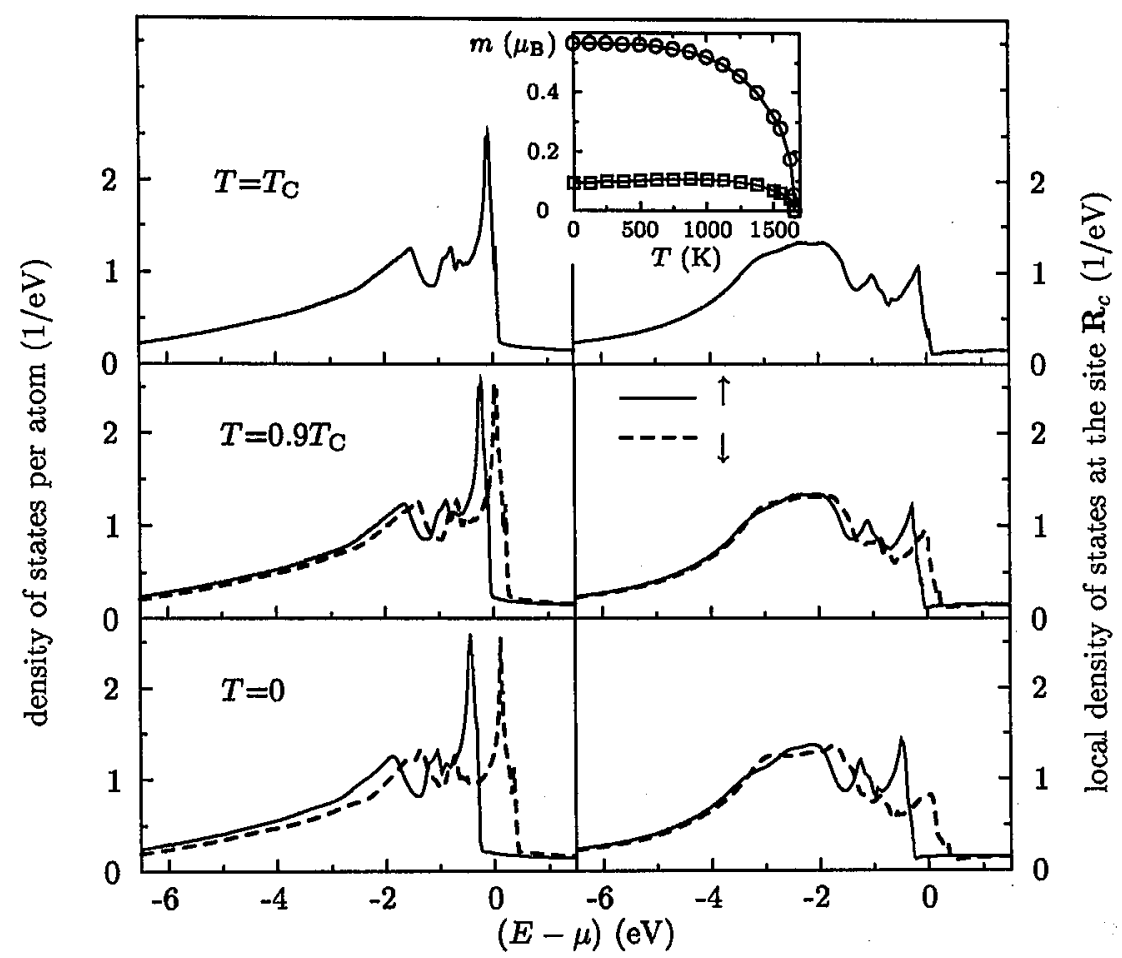

Fig. 1. Density of states (left) and local density of states at the site $\boldsymbol{R}_{c}$, where the core hole was created (right) for three temperatures. Solid lines: majority $(\uparrow)$ spectrum. Dashed lines: minority $(\downarrow)$ spectrum. Inset: magnetisation (circles) and magnetic moment at $\boldsymbol{R}_{c}$ (squares) as functions of temperature $T$.

potential. The corresponding Coulomb-matrix elements are taken to be orbitally independent $\left(U_{L}^{c}=U_{\mathrm{c}}\right)$. We assume an infinite lifetime of the core hole in the initial state for the Auger process as there are no decay terms in the Hamiltonian. Thus, the core-level occupation is a good quantum number. Thermodynamic averages in the presence of the core hole have to be performed in the subspace of the Hilbert space that is built up by all many-body states with a core hole at the site $\boldsymbol{R}_{\mathrm{c}}$. In practice, this is done by introducing an appropriate Lagrange parameter.

The extended Hamiltonian and the averaging procedure introduce two new terms in the valence-band self-energy. The Hartree-like term $-\delta_{\boldsymbol{R} \boldsymbol{R}_{\mathrm{c}}} U_{\mathrm{c}}$ represents the additional core-hole potential seen by the valence electrons. Thereby, the translational symmetry is broken and all occupation numbers become site dependent. Since the correlation effects among the valence electrons depend on the occupations, this introduces an extra screening term in the self-energy.

These screening effects are in general extended over some shells around $\boldsymbol{R}_{\mathrm{c}}$. A reasonable approximation for $3 d$-transition metals is to assume a cmplete screening of the core-hole potential already at the site $\boldsymbol{R}_{\mathrm{c}}$, i.e. the total occupation at $\boldsymbol{R}_{\mathrm{c}}$ is increased by 1 electron, and one is left with a single-site scattering problem 
(for a more detailed discussion see Ref. [6]). Here, the complete screening is used as a condition to fix the core-valence interaction parameter $U_{\mathrm{c}}$ at $T=0$. We find the value $U_{\mathrm{c}}=1.81 \mathrm{eV}$.

The local density of states at the site $\boldsymbol{R}_{\mathrm{c}}$ in the presence of the core hole is shown on the r.h.s. of Fig. 1. The structure of the spectrum has remarkably changed. Spectral weight is transferred to lower energies. Especially for $\downarrow$ electrons a redistribution from energies above to below the chemical potential $\mu$ is visible. By comparing the quasi-particle weights (band-width renormalisation) with the unscreened case, we find the screened case to behave less correlated since here one is closer to the limit of the completely filled $(3 d)$ band. As a consequence of the fact that $\mathrm{Ni}$ is a strong ferromagnet, only minority spin states can be populated to screen the core hole. Indeed this leads to a drastic reduction of the local magnetic moment $\left(0.095 \mu_{\mathrm{B}}\right)$. The temperature dependence of the local magnetic moment in the presence of the core hole is shown in the inset (squares).

We conclude that the comparatively small spin polarisation of Auger electrons for $\mathrm{Ni}[1]$ is due to the screening of the core-hole potential in the initial state. Within the considered itinerant-electron model including $4 s$ and $4 p$ states, however, even a complete screening does not lead to fully vanishing local magnetic moment.

Financial support of the Deutsche Forschungsgemeinschaft within the project No. $158 / 5-1$ is gratefully acknowledged. The numerical calculations were performed on a CrayT3E at the Konrad-Zuse-Zentrum für Informationstechnologie Berlin (ZIB).

\section{References}

[1] R. Allenspach, D. Mauri, M. Taborelli, M. Landolt, Phys. Rev. B 35, 4801 (1987).

[2] J.J. Lander, Phys. Rev. 91, 1382 (1953).

[3] D.A. Papaconstantopoulos, Handbook of the Band Structure of Elemental Solids Plenum, New York 1986.

[4] S. Sugano, Y. Tanabe, H. Kamimura, Multiplets of Transition-Metal Ions in Crystals, in series Pure and Applied Physics, Vol. 33, Academic, New York 1970.

[5] V.I. Anisimov, F. Aryasetiawan, A.I. Lichtenstein, J. Phys., Condens. Matter 9, 767 (1997).

[6] T. Wegner, M. Potthoff, W. Nolting, to be published.

[7] M.M. Steiner, R.C. Albers, L.J. Sham, Phys. Rev. B 45, 13272 (1992).

[8] S. Hirooka, M. Shimizu, Phys. Lett. A 46, 209 (1973).

[9] M.B. Stearns, in: Landoldt-Börnstein, New Series of Group III, Vol. 19a, Ed. H.P.J. Wijn, Springer, Berlin 1984, ch. Magnetic Properties of Metals.

[10] C.-O. Almbladh, L. Hedin, in: Handbook on Synchrotron Radiation, Vol. 1b, Ed. E.-E. Koch, North-Holland, Amsterdam 1983, p. 607. 